\title{
CONVOLUTION OF FUNCTIONALS OF DISCRETE-TIME NORMAL MARTINGALES
}

\author{
QI HAN, CAISHI WANG ${ }^{\bowtie}$ and YULAN ZHOU
}

(Received 27 September 2011)

\begin{abstract}
Let $M=(M)_{n \in \mathbb{N}}$ be a discrete-time normal martingale satisfying some mild requirements. In this paper we show that through the full Wiener integral introduced by Wang et al. ('An alternative approach to Privault's discrete-time chaotic calculus', J. Math. Anal. Appl. 373 (2011), 643-654), one can define a multiplication-type operation on square integrable functionals of $M$, which we call the convolution. We examine algebraic and analytical properties of the convolution and, in particular, we prove that the convolution can be used to represent a certain family of conditional expectation operators associated with $M$. We also present an example of a discrete-time normal martingale to show that the corresponding convolution has an integral representation.
\end{abstract}

2010 Mathematics subject classification: primary 60H40; secondary 46E30.

Keywords and phrases: normal martingale, functional, convolution.

\section{Introduction}

In recent years, there has been much interest in functionals of stochastic processes in discrete time. In 2001 Émery [2] discussed the chaotic representation property of a class of discrete-time stochastic processes. In 2008 Privault [5] surveyed his discretetime chaotic calculus, which is a Malliavin-type theory of stochastic calculus for functionals of discrete-time normal martingales. In 2010 Nourdin et al. [4] considered Rademacher functionals by using Stein's method. Recently Wang et al. [6] introduced a notion of quantum Bernoulli noises and defined corresponding quantum stochastic integrals, which are actually about operator processes acting on functionals of discretetime Bernoulli noises. More recently Wang et al. [7] have presented an alternative approach to Privault's discrete-time chaotic calculus.

Let $M=(M)_{n \in \mathbb{N}}$ be a discrete-time normal martingale satisfying some mild requirements. In [7] the authors introduced a Wiener-type integral with respect to the noise associated with $M$, which is called the full Wiener integral. In this paper

The authors are supported by National Natural Science Foundation of China (Grant No. 11061032) and Natural Science Foundation of Gansu Province (Grant No. 0710RJZA106). The second author is also supported partially by a grant from Northwest Normal University (Grant No. NWNU-KJCXGC-03-61).

(c) 2011 Australian Mathematical Publishing Association Inc. 0004-9727/2011 \$16.00 
we show that through the full Wiener integral, one can define a multiplication-type operation on square integrable functionals of $M$, which we call the convolution. We examine algebraic and analytical properties of the convolution and, in particular, we prove that the convolution can be used to represent a certain family of conditional expectation operators associated with $M$. We also present an example of discretetime normal martingale to show that the corresponding convolution has an integral representation.

The paper is organised as follows. Section 2 recalls some basic notions and facts such as discrete-time normal martingales, the full Wiener integral, and the chaotic representation property. Sections 3 and 4 state our main results. We first define the convolution on square integrable functionals of a discrete-time normal martingale $M$. Then we examine its algebraic and analytical properties and show its interesting link with a certain family of conditional expectation operators associated with $M$. Finally, we present an example of a discrete-time normal martingale to show that the corresponding convolution has an integral representation.

Notation and conventions. Let $\mathbb{N}$ be the set of all nonnegative integers. For a subset $S \subset \mathbb{N}$, we define $\Gamma(S)$ as the finite power set of $S$, namely

$$
\Gamma(S)=\{\sigma \mid \sigma \subset S \text { and } \# \sigma<\infty\},
$$

where $\# \sigma$ means the cardinality of $\sigma$ as a set. If $S=\{0,1, \ldots, k\}$, then we simply write $\Gamma_{k]}=\Gamma(S)$. We set $\Gamma_{-1]}=\Gamma(\emptyset)$, where $\emptyset$ denotes the empty set.

We write $\Gamma=\Gamma(\mathbb{N})$ for brevity. (Clearly, $\Gamma$ is countable.) As usual, $l^{2}(\Gamma)$ denotes the space of square summable real-valued functions on $\Gamma$.

\section{Normal martingale}

Let $(\Omega, \mathcal{F}, P)$ be a probability space with $\mathbb{E}$ denoting the expectation with respect to $P$. We use $\mathcal{L}^{2}(\Omega)$ to mean $\mathcal{L}^{2}(\Omega, \mathcal{F}, P)$ if there is no risk of confusion.

Definition 2.1. An $L^{2}$-stochastic process $M=\left(M_{n}\right)_{n \in \mathbb{N}}$ on $(\Omega, \mathcal{F}, P)$ is called a discrete-time normal martingale if it satisfies:

(i) $\quad \mathbb{E}\left[M_{0} \mid \mathcal{F}_{-1}\right]=0$ and $\mathbb{E}\left[M_{n} \mid \mathcal{F}_{n-1}\right]=M_{n-1}$ for $n \geq 1$;

(ii) $\mathbb{E}\left[M_{0}^{2} \mid \mathcal{F}_{-1}\right]=1$ and $\mathbb{E}\left[M_{n}^{2} \mid \mathcal{F}_{n-1}\right]=M_{n-1}^{2}+1$ for $n \geq 1$,

where $\mathcal{F}_{-1}=\{\emptyset, \Omega\}$ and $\mathcal{F}_{n}=\sigma\left(M_{k} ; 0 \leq k \leq n\right)$ for $n \in \mathbb{N}$.

Let $M=\left(M_{n}\right)_{n \in \mathbb{N}}$ be a discrete-time normal martingale. Then, from $M$, we can construct another stochastic process $Z=\left(Z_{n}\right)_{n \in \mathbb{N}}$ as follows:

$$
Z_{0}=M_{0}, \quad Z_{n}=M_{n}-M_{n-1}, \quad n \geq 1 .
$$

We may view $Z$ as a noise in discrete time, which we call the noise associated with $M$. It can be verified that, as a process on $(\Omega, \mathcal{F}, P), Z$ admits the following two properties:

(i) for each $n \in \mathbb{N}, Z_{n}$ is conditionally centred, that is,

$$
\mathbb{E}\left[Z_{n} \mid \mathcal{F}_{n-1}\right]=0
$$


(ii) for each $n \in \mathbb{N}, Z_{n}$ has a standard conditional quadratic variation, that is,

$$
\mathbb{E}\left[Z_{n}^{2} \mid \mathcal{F}_{n-1}\right]=1
$$

Here $\mathcal{F}_{n}$ is the same as in Definition 2.1.

Recall that $\Gamma$ is the finite power set of $\mathbb{N}$. The next lemma shows that, from the noise $Z$, one can construct an orthonormal system for $\mathcal{L}^{2}(\Omega)$, which is indexed by $\sigma \in \Gamma$.

Lemma 2.2. Let $Z_{\emptyset}=1$, where $\emptyset$ denotes the empty set and

$$
Z_{\sigma}=\prod_{i \in \sigma} Z_{i}, \quad \sigma \in \Gamma, \sigma \neq \emptyset .
$$

Then the set $\left\{Z_{\sigma} \mid \sigma \in \Gamma\right\}$ forms a countable orthonormal system of $\mathcal{L}^{2}(\Omega)$.

For a proof of this lemma, we refer to [2, 5] or [7]. Using this lemma and related general results in functional analysis [1], we come to the next lemma.

Lemma 2.3. There exists a unique isometry $\mathbb{J}: l^{2}(\Gamma) \rightarrow \mathcal{L}^{2}(\Omega)$ such that

$$
\mathbb{J}(f)=\sum_{\sigma \in \Gamma} f(\sigma) Z_{\sigma}, \quad f \in l^{2}(\Gamma),
$$

where the series is convergent in the norm of $\mathcal{L}^{2}(\Omega)$.

The isometry $\mathbb{J}$ mentioned in Lemma 2.3 is referred to as the full Wiener integral operator [7] and $\mathbb{J}(f)$ the full Wiener integral of $f$.

Definition 2.4. The noise $Z$ is said to have the chaotic representation property if the set $\left\{Z_{\sigma} \mid \sigma \in \Gamma\right\}$ is total in $\mathcal{L}^{2}(\Omega)$.

So if the noise $Z$ has a chaotic representation property, the set $\left\{Z_{\sigma} \mid \sigma \in \Gamma\right\}$ actually forms an orthonormal basis of $\mathcal{L}^{2}(\Omega)$. In that case, the full Wiener integral operator $\mathbb{J}: l^{2}(\Gamma) \rightarrow \mathcal{L}^{2}(\Omega)$ becomes an isometric isomorphism.

LEMma 2.5 [7]. Let the noise $Z$ have the chaotic representation property. Then for each $k \in \mathbb{N}$, there exists a bounded operator $\partial_{k}$ on $\mathcal{L}^{2}(\Omega)$ such that

$$
\partial_{k} Z_{\sigma}=\mathbf{1}_{\sigma}(k) Z_{\sigma \backslash k}, \quad \sigma \in \Gamma,
$$

where $\sigma \backslash k$ stands for $\sigma \backslash\{k\}$.

The operator $\partial_{k}$ is called the annihilation operator at $k$ and its dual $\partial_{k}^{*}$ the creation operator. As its name suggests, the creation operator has the following property:

$$
\partial_{k}^{*} Z_{\sigma}=\left(1-\mathbf{1}_{\sigma}(k)\right) Z_{\sigma \cup k}, \quad \sigma \in \Gamma,
$$

where $\sigma \cup k$ means $\sigma \cup\{k\}$. See [7] for details about annihilation and creation operators. 


\section{Convolution}

In this section, we always assume that $M=\left(M_{n}\right)_{n \in \mathbb{N}}$ is a given discrete-time normal martingale on the probability space $(\Omega, \mathcal{F}, P)$. We also assume that the noise $Z=$ $\left(Z_{n}\right)_{n \in \mathbb{N}}$ associated with $M$ has a chaotic representation property (see (2.1) for the meaning of $Z_{n}$ ).

So the set $\left\{Z_{\sigma} \mid \sigma \in \Gamma\right\}$ forms an orthonormal basis of $\mathcal{L}^{2}(\Omega)$, where $Z_{\sigma}$ is defined by (2.3). This means that $\mathcal{F}$ is generated by the noise $Z$ (equivalently, by the normal martingale $M)$. Thus we may call random variables on $(\Omega, \mathcal{F}, P)$ functionals of the normal martingale $M$ or functionals of the noise $Z$.

Note that the full Wiener integral operator $\mathbb{J}: l^{2}(\Gamma) \mapsto \mathcal{L}^{2}(\Omega)$ is an isometric isomorphism (see (2.4) for its definition) and $l^{2}(\Gamma)$ forms an algebra with the usual product given by

$$
(f g)(\sigma)=f(\sigma) g(\sigma), \quad \sigma \in \Gamma,
$$

where $f, g \in l^{2}(\Gamma)$. In view of these two facts, we come to the next definition.

Definition 3.1. Let $\xi, \eta \in \mathcal{L}^{2}(\Omega)$. Then the convolution $\xi * \eta$ of $\xi$ and $\eta$ is defined as

$$
\xi * \eta=\mathbb{I}(f g),
$$

where $f=\mathbb{J}^{-1}(\xi)$ and $g=\mathbb{J}^{-1}(\eta)$.

Thus we have an operation $*$ on $\mathcal{L}^{2}(\Omega)$, which we call the convolution. The next two propositions show that, with the convolution as multiplication, $\mathcal{L}^{2}(\Omega)$ becomes a commutative Banach algebra.

Proposition 3.2. Let $\xi, \eta, \zeta \in \mathcal{L}^{2}(\Omega)$ and $s, t \in \mathbb{R}$ (the real numbers). Then:

(i) $\xi * \eta=\eta * \xi$;

(ii) $\xi *(\eta * \zeta)=(\xi * \eta) * \zeta$;

(iii) $\xi *(s \eta+t \zeta)=s(\xi * \eta)+t(\xi * \zeta)$.

Proof. The proof is straightforward.

Proposition 3.3. The convolution is continuous with respect to the norm of $\mathcal{L}^{2}(\Omega)$; more precisely,

$$
\|\xi * \eta\| \leq\|\xi\|\|\eta\|, \quad \xi, \eta \in \mathcal{L}^{2}(\Omega)
$$

where $\|\cdot\|$ denotes the $\mathcal{L}^{2}(\Omega)$-norm.

Proof. Take $f, g \in l^{2}(\Gamma)$ such that $\xi=\mathbb{J}(f)$ and $\eta=\mathbb{J}(g)$. Then, by the isometric property of $\mathbb{J}$,

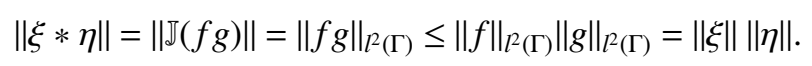

This completes the proof. 
Proposition 3.4. Let $\xi \in \mathcal{L}^{2}(\Omega)$. Then for each $\sigma \in \Gamma$,

$$
\xi * Z_{\sigma}=\left\langle\xi, Z_{\sigma}\right\rangle Z_{\sigma}
$$

where $\left\langle\xi, Z_{\sigma}\right\rangle=\mathbb{E}\left[\xi Z_{\sigma}\right]$.

Proof. Take $f \in l^{2}(\Gamma)$ such that $\xi=\mathbb{J}(f)$. Then, noticing that $Z_{\sigma}=\mathbb{I}\left(\mathbf{1}_{\{\sigma\}}\right)$,

$$
\xi * Z_{\sigma}=\mathbb{J}\left(f \mathbf{1}_{\{\sigma\}}\right)=\sum_{\tau \in \Gamma} f(\tau) \mathbf{1}_{\{\sigma\}}(\tau) Z_{\tau}=f(\sigma) Z_{\sigma},
$$

which together with $\left\langle\xi, Z_{\sigma}\right\rangle=f(\sigma)$ gives (3.1).

In the following we write $\mathcal{F}_{-1}=\{\emptyset, \Omega\}$ and $\mathcal{F}_{n}=\sigma\left(M_{k} ; 0 \leq k \leq n\right)$ for $n \in \mathbb{N}$. In this way $\left(\mathcal{F}_{n}\right)_{n \geq-1}$ forms a filtration on $(\Omega, \mathcal{F}, P)$. We note that $\mathcal{F}_{n}$ can also be expressed in terms of $Z$, namely $\mathcal{F}_{n}=\sigma\left(Z_{k} ; 0 \leq k \leq n\right)$.

For $k \in \mathbb{N}$, we define a functional $\psi_{k}$ as

$$
\psi_{k}=\sum_{\sigma \in \Gamma_{k]}} Z_{\sigma}
$$

where $\Gamma_{k]}=\{\sigma \mid \sigma \subset\{0,1, \ldots, k\}\}$. Clearly $\psi_{k} \in \mathcal{L}^{2}(\Omega)$ for each $k \in \mathbb{N}$. The next proposition is one of our main results, showing that the conditional expectation operator $\mathbb{E}\left[\cdot \mid \mathcal{F}_{k}\right]$ can be represented by $\psi_{k}$ through the convolution.

Proposition 3.5. Let $\xi \in \mathcal{L}^{2}(\Omega)$ and $k \in \mathbb{N}$. Then

$$
\mathbb{E}\left[\xi \mid \mathcal{F}_{k}\right]=\xi * \psi_{k}
$$

Proof. We first show that $\mathbb{E}\left[Z_{\sigma} \mid \mathcal{F}_{k}\right]=0$ if $\sigma \in \Gamma \backslash \Gamma_{k]}$. In fact, if $\sigma \in \Gamma \backslash \Gamma_{k]}$, then $\sigma \neq \emptyset$ and $n=\max \sigma>k$; hence, by the conditionally centred property of $Z_{n}$ (see (2.2)),

$$
\mathbb{E}\left[Z_{\sigma} \mid \mathcal{F}_{k}\right]=\mathbb{E}\left[Z_{\sigma \backslash n} \mathbb{E}\left[Z_{n} \mid \mathcal{F}_{n-1}\right] \mid \mathcal{F}_{k}\right]=0 .
$$

We now use this property to verify (3.3). Let $\xi=\mathbb{J}(f)$ with $f \in l^{2}(\Gamma)$. Then

$$
\mathbb{E}\left[\xi \mid \mathcal{F}_{k}\right]=\sum_{\sigma \in \Gamma_{k]}} f(\sigma) \mathbb{E}\left[Z_{\sigma} \mid \mathcal{F}_{k}\right]+\sum_{\sigma \in \Gamma \backslash \Gamma_{k]}} f(\sigma) \mathbb{E}\left[Z_{\sigma} \mid \mathcal{F}_{k}\right]=\sum_{\sigma \in \Gamma_{k]}} f(\sigma) Z_{\sigma} .
$$

On the other hand, by Proposition 3.4, we find that

$$
\xi * \psi_{k}=\sum_{\sigma \in \Gamma_{k]}} \xi * Z_{\sigma}=\sum_{\sigma \in \Gamma_{k]}}\left\langle\xi, Z_{\sigma}\right\rangle Z_{\sigma}=\sum_{\sigma \in \Gamma_{k]}} f(\sigma) Z_{\sigma} .
$$

Thus (3.3) holds.

The next proposition suggests that the sequence $\psi_{k}, k \in \mathbb{N}$, can be viewed as an approximate identity of the Banach algebra $\left(\mathcal{L}^{2}(\Omega), *\right)$. 
Proposition 3.6. Let $\xi \in \mathcal{L}^{2}(\Omega)$. Then

$$
\lim _{k \rightarrow \infty}\left\|\xi * \psi_{k}-\xi\right\|=0 .
$$

Proof. Set $\xi_{k}=\xi * \psi_{k}, k \in \mathbb{N}$. Then it follows from Proposition 3.5 that

$$
\xi_{k}=\mathbb{E}\left[\xi \mid \mathcal{F}_{k}\right], \quad k \in \mathbb{N} .
$$

It is easy to see that $\mathcal{F}=\sigma\left(\bigcup_{k \in \mathbb{N}} \mathcal{F}_{k}\right)$. Thus by the well-known martingale convergence theorem (see, for example, [3]) we come to (3.4).

As an immediate consequence of Proposition 3.5, we have the following version of the Clark formula in discrete time (see, for example, [7]).

Corollary 3.7. For each $\xi \in \mathcal{L}^{2}(\Omega)$,

$$
\xi=\mathbb{E} \xi+\sum_{k \in \mathbb{N}} Z_{k} \partial_{k}\left(\xi * \psi_{k}\right)=\mathbb{E} \xi+\sum_{k \in \mathbb{N}} Z_{k}\left[\left(\partial_{k} \xi\right) * \psi_{k-1}\right],
$$

where $\psi_{k}$ is defined by (3.2).

\section{Integral representation}

In this section, we present an example of a discrete-time normal martingale to show that the corresponding convolution has an integral representation.

Let $\Omega=\{-1,1\}^{\mathbb{N}}$, the set of all mappings $\omega: \mathbb{N} \rightarrow\{-1,1\}$. Then $\Omega$ is a commutative group with the natural product given by

$$
\left(\omega_{1} \omega_{2}\right)(n)=\omega_{1}(n) \omega_{2}(n), \quad n \in \mathbb{N},
$$

where $\omega_{1}, \omega_{2} \in \Omega$. Note that this group has 1 as its identity and, moreover, each $\omega \in \Omega$ has itself as its inverse, namely $\omega^{-1}=\omega$.

Let $\left(Z_{n}\right)_{n \in \mathbb{N}}$ be the sequence of canonical projections on $\Omega$ given by

$$
Z_{n}(\omega)=\omega(n), \quad \omega \in \Omega
$$

Denote by $\mathcal{F}$ the $\sigma$-field generated by the sequence $\left(Z_{n}\right)_{n \in \mathbb{N}}$. Then (see [5]) there exists a unique probability measure $P$ on $\mathcal{F}$ such that

$$
P \circ\left(Z_{n_{1}}, Z_{n_{2}}, \ldots, Z_{n_{k}}\right)^{-1}\left\{\left(\epsilon_{1}, \epsilon_{2}, \ldots, \epsilon_{k}\right)\right\}=\frac{1}{2^{k}}
$$

for $n_{j} \in \mathbb{N}, \epsilon_{j} \in\{-1,1\}(1 \leq j \leq k)$ with $n_{i} \neq n_{j}$ when $i \neq j$ and $k \in \mathbb{N}$ with $k \geq 1$. Note that $P$ is also the only invariant probability measure on the group $\Omega$.

So we come to a probability measure space $(\Omega, \mathcal{F}, P)$ and a sequence $Z=\left(Z_{n}\right)_{n \in \mathbb{N}}$ of independent random variables on it. Define

$$
M_{n}=\sum_{k=0}^{n} Z_{k}, \quad n \in \mathbb{N} .
$$


Then $M=\left(M_{n}\right)_{n \in \mathbb{N}}$ is a discrete-time normal martingale on $(\Omega, \mathcal{F}, P)$. Thus $Z$ is the noise associated with $M$, which we call the Bernoulli noise. It can be shown [5] that the Bernoulli noise $Z$ has the chaotic representation property.

Proposition 4.1. Let $\xi, \quad \eta \in \mathcal{L}^{2}(\Omega)$. Then the convolution $\xi * \eta$ defined as in Definition 3.1 has the following integral representation:

$$
\xi * \eta\left(\omega_{1}\right)=\int_{\Omega} \xi(\omega) \eta\left(\omega_{1} \omega\right) d P(\omega), \quad \text { for P-almost all } \omega_{1} \in \Omega .
$$

Proof. Define an operation $\diamond$ on $\mathcal{L}^{2}(\Omega)$ as follows:

$$
\xi \diamond \eta\left(\omega_{1}\right)=\int_{\Omega} \xi(\omega) \eta\left(\omega_{1} \omega\right) d P(\omega), \quad \omega_{1} \in \Omega
$$

where $\xi, \eta \in \mathcal{L}^{2}(\Omega)$. It can be shown that $\xi \diamond \eta \in \mathcal{L}^{2}(\Omega)$ whenever $\xi, \eta \in \mathcal{L}^{2}(\Omega)$ and, moreover, $\mathcal{L}^{2}(\Omega)$ becomes a commutative Banach algebra with the operation $\diamond$ as multiplication.

Now let $\xi, \eta \in \mathcal{L}^{2}(\Omega)$. To complete the proof, we need only verify that $\xi * \eta=\xi \diamond \eta$. Take $g \in l^{2}(\Gamma)$ such that

$$
\eta=\sum_{\sigma \in \Gamma} g(\sigma) Z_{\sigma}
$$

For each $\sigma \in \Gamma$, noticing that $Z_{\sigma}\left(\omega \omega_{1}\right)=Z_{\sigma}(\omega) Z_{\sigma}\left(\omega_{1}\right), \omega, \omega_{1} \in \Omega$, we have

$$
\xi \diamond Z_{\sigma}\left(\omega_{1}\right)=\int_{\Omega} \xi(\omega) Z_{\sigma}\left(\omega_{1} \omega\right) d P(\omega)=Z_{\sigma}\left(\omega_{1}\right) \int_{\Omega} \xi(\omega) Z_{\sigma}(\omega) d P(\omega), \quad \omega_{1} \in \Omega,
$$

which together with Proposition 3.4 gives

$$
\xi * Z_{\sigma}=\xi \diamond Z_{\sigma}
$$

Thus by the continuity of both $*$ and $\diamond$ we get

$$
\xi * \eta=\sum_{\sigma \in \Gamma} g(\sigma) \xi * Z_{\sigma}=\sum_{\sigma \in \Gamma} g(\sigma) \xi \diamond Z_{\sigma}=\xi \diamond \eta
$$

This completes the proof.

\section{References}

[1] J. B. Conway, A Course in Functional Analysis, 2nd edn (Springer, New York, 1990).

[2] M. Émery, 'A discrete approach to the chaotic representation property', in: Séminaire de Probabilités, XXXV, Lecture Notes in Mathematics, 1755 (Springer, Berlin, 2001), pp. 123-138.

[3] O. Kallenberg, Foundations of Modern Probability (Springer, Berlin, 1997).

[4] I. Nourdin, G. Peccati and G. Reinert, 'Stein's method and stochastic analysis of Rademacher functionals', Electron. J. Probab. 15 (2010), 1703-1742.

[5] N. Privault, 'Stochastic analysis of Bernoulli processes', Probab. Surv. 5 (2008), 435-483. 
[6] C. S. Wang, H. F. Chai and Y. C. Lu, 'Discrete-time quantum Bernoulli noises', J. Math. Phys. 51 (2010), 053528.

[7] C. S. Wang, Y. C. Lu and H. F. Chai, 'An alternative approach to Privault's discrete-time chaotic calculus', J. Math. Anal. Appl. 373 (2011), 643-654.

QI HAN, School of Mathematics and Information Science,

Northwest Normal University, Lanzhou, Gansu 730070, PR China

e-mail: hanqi1978@nwnu.edu.cn

CAISHI WANG, School of Mathematics and Information Science, Northwest Normal University, Lanzhou, Gansu 730070, PR China e-mail: wangcs@nwnu.edu.cn, cswangnwnu@163.com

YULAN ZHOU, School of Mathematics and Information Science, Northwest Normal University, Lanzhou, Gansu 730070, PR China e-mail: zhouylw123@163.com 